Article

\title{
Deep Electrical Modulation of Terahertz Wave Based on Hybrid Metamaterial-Dielectric-Graphene Structure
}

\author{
Liangping Xia ${ }^{1,2, *}$, Xin Zhang ${ }^{2}$, Man Zhang ${ }^{3}$, Suihu Dang ${ }^{1}$, Shijian Huang ${ }^{1}$, Yong Tan ${ }^{1}$, \\ Wenjuan Yan ${ }^{1}$ and Hong-Liang Cui ${ }^{2}$ \\ 1 School of Electronic Information Engineering, Yangtze Normal University, Chongqing 408100, China; \\ dangsuihu@yznu.cn (S.D.); huangshijian@yznu.cn (S.H.); cquty@126.com (Y.T.); 20000032@yznu.cn (W.Y.) \\ 2 Chongqing Key laboratory of Multi-Scale manufacturing Technology, Chongqing Institute of Green and \\ Intelligent Technology, Chinese Academy of Sciences, Chongqing 400714, China; zx371788177@sina.com (X.Z.); \\ hlcui2012@126.com (H.-L.C.) \\ 3 Institute of Optics and Electronics, Chinese Academy of Sciences, Chengdu 610209, China; \\ zhangman@ioe.ac.cn \\ * Correspondence: xialp@cigit.ac.cn; Tel.: +00862372792166
}

Received: 6 January 2019; Accepted: 29 January 2019; Published: 1 February 2019

\begin{abstract}
A terahertz modulation structure based on hybrid metamaterial and graphene is proposed and demonstrated in this work. The metamaterial with a square slit ring array excites terahertz resonance in the slits and enhances the interaction between the terahertz wave and graphene. The graphene layer acting as the active material is tuned by the applied electrical field. With the separation by a dielectric layer between the graphene and the metallic structure, the resonant frequency and transmitted energy are both modulated by the graphene. Experimental result indicates that the modulation depth of the terahertz transmitted amplitude is $65.1 \%$ when the applied modulation voltage is tuned $5 \mathrm{~V}$.
\end{abstract}

Keywords: terahertz; modulation; graphene; metamaterial

\section{Introduction}

Terahertz $(\mathrm{THz})$ is the electromagnetic wave of the frequency band between microwave and infrared light. It is a research hotspot in the application of penetrating imaging [1], high speed wireless communication [2], and medical sensing [3]. Modulation of wave amplitude is one of the basic operations in its applications [4-6]. Traditional modulation methods are based on conventional semiconductor materials or mechanical scanning $[7,8]$. However, the modulation speed is limited with these methods due to low carrier mobility (e.g., $\sim 1400 \mathrm{~cm}^{2} \mathrm{~V}^{-1} \mathrm{~s}^{-1}$ for Si [9]) or constraints on mechanical motion.

Graphene is a two dimensional material with carrier mobility $\left(\sim 2 \times 10^{5} \mathrm{~cm}^{2} \mathrm{~V}^{-1} \mathrm{~s}^{-1}\right.$ [10] $)$ about one order of magnitude higher than that of semiconductors [11]. The Fermi level of graphene could be electrically tuned [12]. This property makes graphene hugely popular in making tunable devices, such as absorbers [13], detectors [14], sensors [15], and modulators [16]. However, the commonly used graphene is with the thickness of a single layer of carbon atoms, which leads to a weak interaction between the electromagnetic wave and the graphene. Metallic metamaterial has the unique property of field enhancement, which has been employed for ultra-sensitive detection [17], super-resolution imaging [18], and high-gain antennas [19]. A combination of graphene and metamaterial to enhance interaction with light has been reported recently [20-22]. It has been demonstrated that by introducing metamaterial into a graphene based $\mathrm{THz}$ modulator, the amplitude modulation depth could be enhanced by $50 \%$ at the resonant frequency of the metamaterial [23]. However, the resonant frequencies 
are not tunable as the graphene was in direct contact with the metallic structures in most reported works, which limits the further increases of the modulation depth.

In this work, a graphene based $\mathrm{THz}$ modulator with the hybrid structure of combined graphene and the metallic slit ring array separated by a dielectric layer is proposed. With resonant excitation in the metallic slit ring, the local $\mathrm{THz}$ field is enhanced by several orders of magnitude, and the interaction between graphene and $\mathrm{THz}$ field is strongly magnified. By inserting a thin layer of dielectric material between the metallic structure and graphene, the resonant frequency is tuned with the modulation voltage synchronously, which leads to a high modulation depth in the transmission wave. The enhancement of the modulation depth in the hybrid structure is discussed theoretically and demonstrated experimentally.

\section{Device Structure}

The configuration of the proposed THz modulation structure is shown in Figure 1a. The hybrid structure is supported by a low loss plastic substrate, on which the first layer deposited is the metallic metamaterial, composed of a periodic structure with the unit cell shown in the schematic drawing of Figure 1a. In the unit cell, the square slit ring with width marked g, period $\mathrm{P}$, and inside length $\mathrm{L}$ are also marked. When $\mathrm{THz}$ wave impinges onto the square slit ring, resonance will be excited and the local field confined in gap of the square slit ring will be enhanced. The second layer is a dielectric with the thickness of $t$ acting as a spacer to avoid direct contact of the graphene and the metallic structure. The graphene film on top of the dielectric layer acts as the active layer. In the electrical modulation process, the metallic metamaterial also plays the role of bottom electrode to apply modulation voltage. With the separation of the dielectric spacer, the applied modulation electrical field is formed between the graphene and the metamaterial. And the conductivity of the graphene is tuned with the applied modulation voltage. It leads to the resonant change in the metamaterial and the transmitted $\mathrm{THz}$ wave is modulated. The modulation depth of the transmitted amplitude is defined as:

$$
M D=\frac{\left|T\left(V_{1}\right)-T\left(V_{2}\right)\right|}{\max \left\{T\left(V_{1}\right), T\left(V_{2}\right)\right\}} \times 100 \%
$$

where the $T\left(V_{1}\right), T\left(V_{2}\right)$ are the transmission at the different modulation voltages.
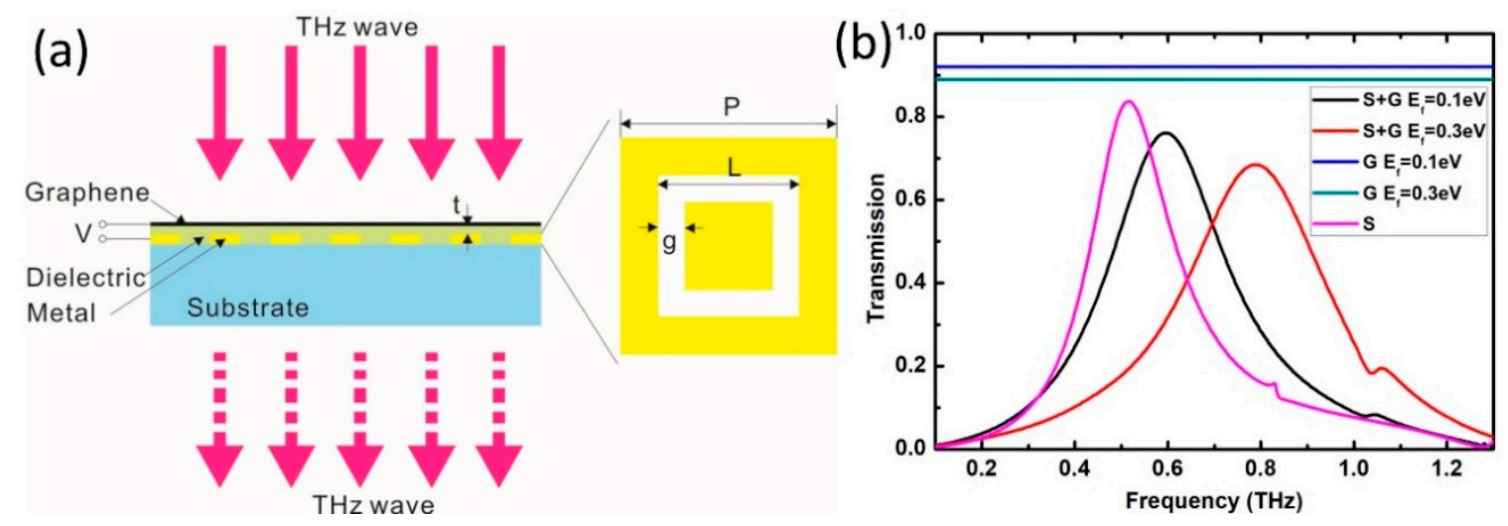

Figure 1. (a) The schematic drawing of the terahertz (THz) modulation structure based on hybrid graphene and metamaterial. (b) The simulated spectrum of the structures with and without the metamaterial in the cases of different Fermi levels.

To demonstrate the modulation enhancement in the hybrid structure, the transmitted $\mathrm{THz}$ spectrum is simulated with the finite element method (FEM). A polyethylene terephthalate (PET) film of thickness $30 \mu \mathrm{m}$ is taken as the low loss plastic substrate. Aluminum is chosen as the material of the metamaterial with $P=150 \mu \mathrm{m}, g=12.5 \mu \mathrm{m}$, and $L=90 \mu \mathrm{m}$. The dielectric material is polymethyl methacrylate (PMMA) with the thickness $t=0.5 \mu \mathrm{m}$ as the spacer between the graphene and the 
metallic structure. The thickness of graphene is set as $1 \mathrm{~nm}$ and the conductivity is calculated with the Drude mode [24]. In this mode, the oscillation frequency $\gamma=2 \mathrm{THz}$.

The transmission spectrum of the hybrid structure at different Fermi level is shown in Figure $1 \mathrm{~b}$. The pink curve is for the pure metallic structure on the substrate, which shows a transmission peak at the frequency of $f_{\text {res }}=0.51 \mathrm{THz}$, which indicates that the resonant is excited by the metallic square slit ring. When the graphene layer is added, the black curve for the $E f=0.1 \mathrm{eV}$ shows that the resonant frequency is blue shifted to $f_{\text {res }}=0.6 \mathrm{THz}$. When the Fermi level of graphene is changed to $E f=0.3 \mathrm{eV}$, the transmission is the red curve. It shows that the transmission peak is blue shifted to $f_{\text {res }}=0.79 \mathrm{THz}$ and the value of the peak is lower. The resonant frequency shift reveals that the dielectric condition is changed when the Fermi level of the graphene is tuned. It leads to a remarkable change of the transmission near the resonant frequency. In comparison, a control sample without the metallic metamaterial is simulated under the same condition. The blue and the green curves are corresponding to the different Fermi level of $0.1 \mathrm{eV}$ and $0.3 \mathrm{eV}$ respectively. The flat transmission lines indicate that there is no resonance in the control structures. And the transmission difference between the two lines is much lower than that of the hybrid structures. When calculating the modulation depth with Formula (1), there is a maximum $M D=67.3 \%$ at the frequency of $\mathrm{f}=0.52 \mathrm{THz}$ for the hybrid structure, while the value of the $M D$ is only $3.3 \%$ for the control structure without the metallic square slit ring. It means that the modulation depth is enhanced more than 20 times in the proposed hybrid structure.

\section{Simulations}

To analyze the resonant conditions and field enhancement in the hybrid structure, the electric field distributions at the frequency of the transmission peak are simulated firstly. When the incident $\mathrm{THz}$ wave is $\mathrm{x}$ polarized, the field distribution in the graphene layer is shown in Figure 2a. It indicates that the electric field is mainly located around the regions of the metallic slits. Along the electric polarization direction, the distribution reveals that the resonant is mainly excited along the side of the square slit. At the same frequency, Figure $2 b$ is the electric field distributions in the $x-z$ plane at the place of the blue dotted line in Figure 2a. It shows that the enhanced field caused by the resonance is located in an area along the $z$ direction. And graphene layer at the placement of $z=0.15 \mu \mathrm{m}$ is immerged in the localized field, which leads to a strong interaction between the graphene and the localized THz field. As a result the modulation of the transmitted $\mathrm{THz}$ wave is enhanced under such strong interactions.
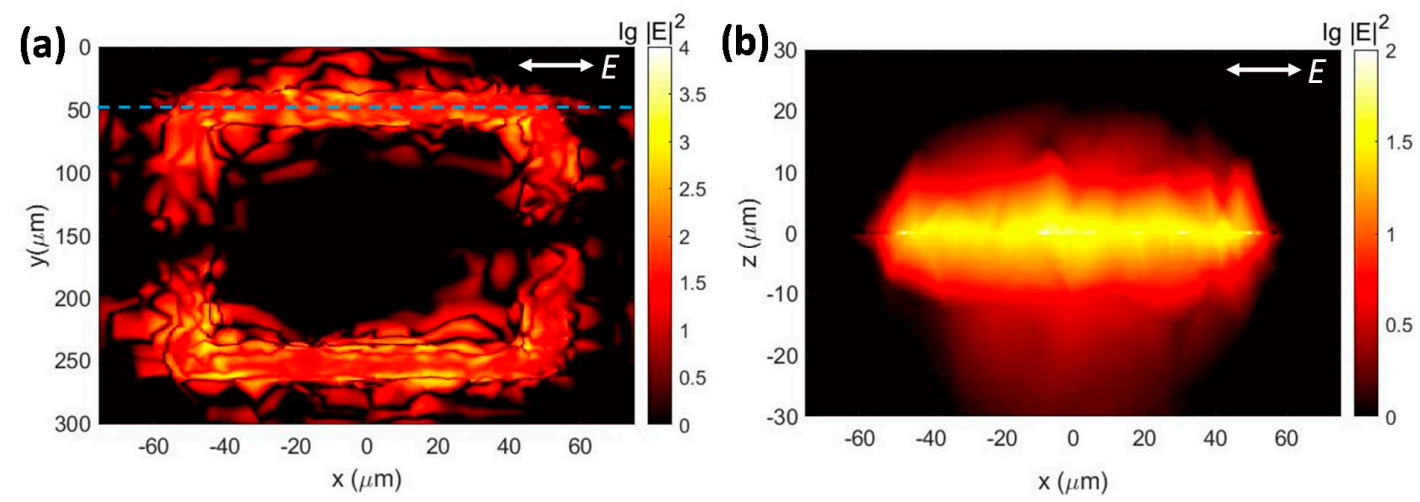

Figure 2. The electrical field distributions: (a) in the graphene layer; (b) in $x-z$ plane.

The influence of the structure parameters on the resonance and the modulation enhancement are further discussed. In the hybrid structure, the side length of the square slit is simulated firstly, and the result is shown in Figure 3a. The black square dots display the relationship of the resonant frequency with the side length of the slit. The black solid fitting line indicates that the resonant frequency is exponentially decaying with the value of L. It illustrates that the resonant frequency decrease is fast, 
which is due to fact that the resonant is mainly excited along the side of the square slit as shown in Figure 2. The blue circular dots are the maximum modulation depth and the blue solid fitting line indicates that the modulation depth is approximately linearly decreasing with the side length. The result reveals that the decrease of the modulation depth is slow, and the minimum $M D$ is larger than $60 \%$. It also reveals that by changing the side length of the square slit, the working frequency of the hybrid structured THz modulator could be tuned in a wideband and the modulation depth could be kept at a high level.
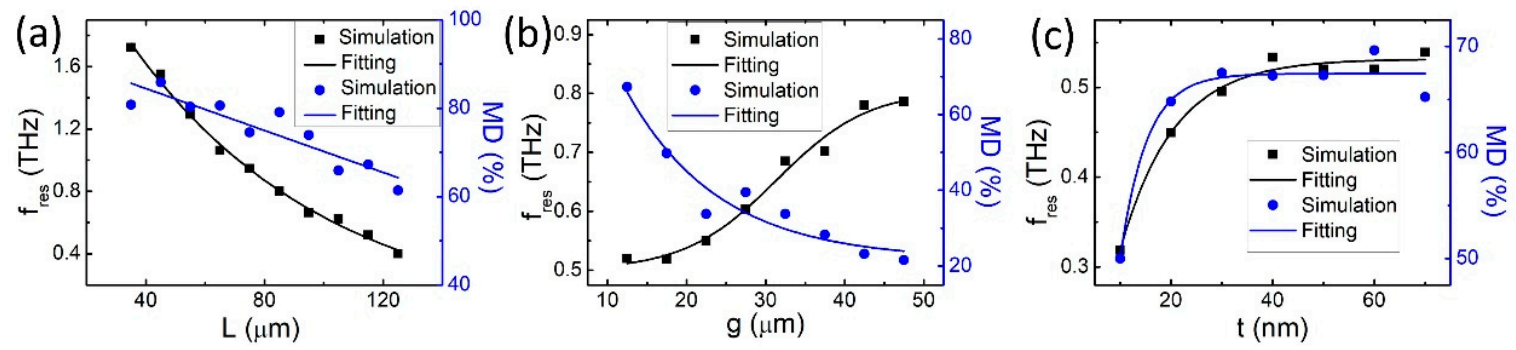

Figure 3. Relationship between resonant frequency and modulation depth with: (a) inner side length; (b) slit gap width of the slit ring; (c) thickness of dielectric layer.

Another key parameter is the slit width in the metallic metamaterial. Its influence on the resonant frequency and modulation depth is calculated and displayed in Figure $3 \mathrm{~b}$. The black square dots and the solid fitting curve show that the resonant frequency increases with the slit width slowly. However, the blue dots and the fitting curve standing for the modulation depth is exponentially decaying with the value of $g$. Further analyzing the transmission spectral curves, reveal that although the resonant frequency is increased with the slit width, the quality factor is getting lower. It is due to the fact that the transmission at the lower frequencies is kept at a high level with no change of the side length of the slit, which leads to a lower modulation depth when the slit width is larger. The results also reveal that a narrower slit is better in the hybrid structured modulator due to stronger confinement of the field, and the stronger interaction with the active graphene layer.

In the hybrid structure, the dielectric layer between the metamaterial and graphene acts as a spacer to avoid the active material directly touching with the metallic layer. When increasing its thickness, as seen in Figure 3c, the resonant frequency and the modulation depth are all increased obviously when the thickness is lower than $20 \mathrm{~nm}$. It is due to the coupling between the graphene layer and the metallic metamaterial layer being much stronger and more sensitive to the separation distance when the latter is relatively short. By further increasing the thickness of the dielectric layer, the resonant frequency and the modulation depth tend to be flat as the coupling between them remain stable. This result reveals that the fabrication tolerance of the dielectric layer could be within a large value in theory. However, a thicker dielectric layer needs a higher modulation voltage to tune the graphene while a thinner dielectric layer is easy to breakdown under the applied voltage in practice.

\section{Measurements}

The proposed modulator based on hybrid structure is fabricated with the following process. Firstly, the Al layer with the thickness of $100 \mathrm{~nm}$ is deposited onto the PET substrate with the thickness of $30 \mu \mathrm{m}$. With photo lithography and wet etching, the square slit ring arrayed structure is fabricated, and its microscopic image is shown in Figure 4a. The photography indicates that the square slits are distributed uniformly. Secondly, the PMMA solution is spin coated onto the metamaterial with the thickness of $50 \mathrm{~nm}$. After drying, the PMMA acts as the dielectric layer to separate the graphene and the metallic square slit ring structure. Then the upper Ag electrode is deposited onto the PMMA and followed by transferring the graphene film grown by chemical vapor deposition (CVD) with wet method [25]. The graphene on the PMMA is partly touching the Ag electrode to connect the applied modulation voltage. 
After fabrication, the device is measured with a THz time-domain spectroscopy (THz-TDS) system (Advanced Photonix, Inc., Michigan, USA). With this equipment, the transmission spectrum at different modulation voltage is obtained and shown in Figure $4 \mathrm{~b}$. The black square dot curve indicates that the resonant is achieved at the frequency of $0.6 \mathrm{THz}$ when the modulation voltage is $-10 \mathrm{~V}$, which is the same as the theoretical result when the Fermi level of the graphene is $0.1 \mathrm{eV}$. By changing the modulation voltage to $-15 \mathrm{~V}$, the circle dot curve indicates that the resonant frequency is tuned to $0.775 \mathrm{THz}$ and the transmission peak is reduced as the theory predicts. The resonant frequency is close to the theoretical result of $E f=0.3 \mathrm{eV}$. The blue star curve standing for the experimental modulation depth is calculated with Formula (1). It indicates that the maximum modulation depth is $65.1 \%$ at the frequency of $0.575 \mathrm{THz}$, which is close to the theoretical result in Figure $1 \mathrm{~b}$. It demonstrates that by separating the graphene layer and the metallic metamaterial with the dielectric layer of PMMA, the resonance of the metamaterial is tuned with the active material. And benefiting from the enhanced interaction between the graphene and the $\mathrm{THz}$ wave in the localized field of the metamaterial, the modulation of the transmitted THz wave is enhanced.

(a)
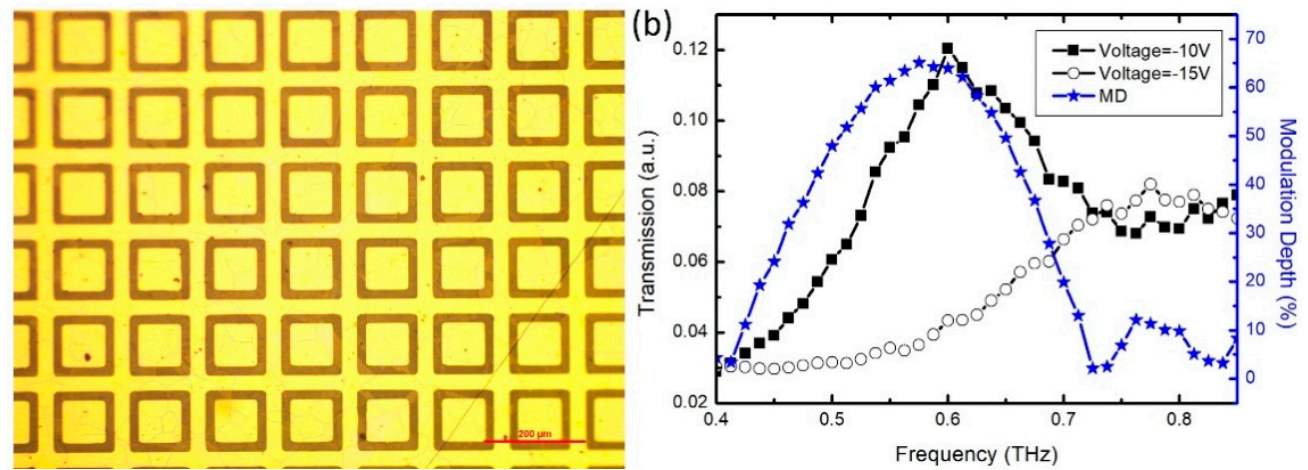

Figure 4. (a) Microscope photography of the fabricated metamaterial. (b) Measured transmission spectrum at different modulation voltage and the corresponding modulation depth.

At last, the characteristic of the THz modulation device enhanced with the metallic square slit array is compared with other graphene based $\mathrm{THz}$ modulation works. The result is shown in Table 1. It indicates that the amplitude $\mathrm{THz}$ modulation depth of the device without the metallic structure enhancement is less than $25 \%$. Comparing with the device with metallic structure directly touching the graphene layer, the modulation depth of the works is close to each other, but the Fermi level tuning requires a wider range for the previous reported work.

Table 1. Performance comparison of graphene based THz modulators. PMMA: polymethyl methacrylate.

\begin{tabular}{cc}
\hline Graphene Based THz Amplitude Modulation & Amplitude Modulation Depth \\
\hline Graphene $/ \mathrm{SiO}_{2} / \mathrm{pSi}[16]$ & $15 \%$ \\
Graphene $/ \mathrm{Al}_{2} \mathrm{O}_{3} / \mathrm{pSi}[26]$ & $22 \%$ \\
Metallic circle ring/Graphene/SiO $/ \mathrm{pSi}[23]$ & $50 \%$ \\
Metallic square ring/PMMA/Graphene/PET (this work) & $65.1 \%$ \\
\hline
\end{tabular}

\section{Conclusions}

In summary, a THz modulator with the hybrid structure of metallic metamaterial and graphene is analyzed theoretically and demonstrated experimentally. Theory and simulation indicate that the resonance is mainly along the side of the square slit ring and lead to the resonant frequency being strongly sensitive to the side length, which could be used to change the working frequency. The narrow slit width with a higher resonant quality factor is beneficial in achieving a high modulation depth. In the theoretical result, the thickness of the dielectric layer has a large tolerance in fabrication. The measured transmission spectrum exhibits the same general tendency as the theory result and the 
amplitude modulation depth of $65.1 \%$ is obtained when the dynamic range of the modulation voltage is $5 \mathrm{~V}$.

Author Contributions: L.X., X.Z., M.Z., S.D., S.H., Y.T., W.Y., and H.-L.C. designed and conceived this study. L.X. and X.Z. performed the experiments. L.X. wrote this paper. All authors approved this manuscript.

Funding: This research was funded by National Natural Science Foundation of China $(61775213,61875196$, 51703227) and Scientific and Technological Research Projects of Chongqing Education Commission (KJ1601211).

Conflicts of Interest: The authors declare no conflict of interest.

\section{References}

1. Federici, J.F.; Schulkin, B.; Huang, F.; Gary, D.; Barat, R.; Oliveira, F.; Zimdars, D. THz imaging and sensing for security applications: Explosives, weapons and drugs. Semicond. Sci. Tech. 2005, 20, S266. [CrossRef]

2. Krumbholz, N.; Gerlach, K.; Rutz, F.; Koch, M.; Piesiewicz, R.; Kürner, T.; Mittleman, D. Omnidirectional terahertz mirrors: A key element for future terahertz communication systems. Appl. Phys. Lett. 2006, 88, 202905. [CrossRef]

3. Fitzgerald, A.J.; Berry, E.; Zinovev, N.N.; Walker, G.C.; Smith, M.A.; Chamberlain, J.M. An introduction to medical imaging with coherent terahertz frequency radiation. Phys. Med. Biol. 2002, 47, R67. [CrossRef] [PubMed]

4. Foschini, G.J.; Golden, G.D.; Valenzuela, R.A.; Wolniansky, P.W. Simplified processing for high spectral efficiency wireless communication employing multi-element arrays. IEEE J. Sel. Area. Commun. 1999, 17, 1841-1852. [CrossRef]

5. Mesleh, R.; Elgala, H.; Haas, H. On the performance of different OFDM based optical wireless communication systems. J. Opt. Commun. Netw. 2011, 3, 620-628. [CrossRef]

6. Cho, K.; Yoon, D. On the general BER expression of one-and two-dimensional amplitude modulations. IEEE Trans. Commun. 2002, 50, 1074-1080.

7. Kleine-Ostmann, T.; Pierz, K.; Hein, G.; Dawson, P.; Koch, M. Audio signal transmission over THz communication channel using semiconductor modulator. Electron. Lett. 2004, 40, 124-126. [CrossRef]

8. Sabah, C. Tunable metamaterial design composed of triangular split ring resonator and wire strip for S-and C-microwave bands. Prog. Electromagn. Res. 2010, 22, 341-357. [CrossRef]

9. Holl, P.; Kemmer, J.; Prechtel, U.; Ziemann, T.; Hauff, D.; Lutz, G.; Schwarz, A. A double-sided silicon strip detector with capacitive readout and a new method of integrated bias coupling. IEEE Trans. Nuc. Sci. 1989, 36, 251-255. [CrossRef]

10. Bolotin, K.I.; Sikes, K.J.; Jiang, Z.; Klima, M.; Fudenberg, G.; Hone, J.; Kim, P.; Stormer, H.L. Ultrahigh electron mobility in suspended graphene. Solid State Commun. 2008, 146, 351-355. [CrossRef]

11. Zhu, Y.; Murali, S.; Cai, W.; Li, X.; Suk, J.W.; Potts, J.R.; Ruoff, R.S. Graphene and graphene oxide: Synthesis, properties, and applications. Adv. Mater. 2010, 22, 3906-3924. [CrossRef] [PubMed]

12. Schwierz, F. Graphene transistors. Nat. Nanotechnol. 2010, 5, 487. [CrossRef] [PubMed]

13. Tan, W.D.; Su, C.Y.; Knize, R.J.; Xie, G.Q.; Li, L.J.; Tang, D.Y. Mode locking of ceramic Nd: Yttrium aluminum garnet with graphene as a saturable absorber. Appl. Phys. Lett. 2010, 96, 031106. [CrossRef]

14. Mueller, T.; Xia, F.; Avouris, P. Graphene photodetectors for high-speed optical communications. Nat.Photonics 2010, 4, 297. [CrossRef]

15. Hill, E.W.; Vijayaragahvan, A.; Novoselov, K. Graphene sensors. IEEE Sens. J. 2011, 11, 3161-3170. [CrossRef]

16. Sensale-Rodriguez, B.; Yan, R.; Kelly, M.M.; Fang, T.; Tahy, K.; Hwang, W.S.; Jena, D.; Liu, L.; Xing, H.G. Broadband graphene terahertz modulators enabled by intraband transitions. Nat. Commun. 2012, 3, 780. [CrossRef]

17. Wang, S.; Xia, L.; Mao, H.; Jiang, X.; Yan, S.; Wang, H.; Wei, D.; Cui, H.L.; Du, C. Terahertz biosensing based on a polarization-insensitive metamaterial. IEEE Photonic. Tech. Lett. 2016, 28, 986-989. [CrossRef]

18. Lu, D.; Liu, Z. Hyperlenses and metalenses for far-field super-resolution imaging. Nat. Commun. 2012, 3, 1205. [CrossRef]

19. Yuan, G.; Dong, X.; Deng, Q.; Liu, C.; Lu, Y.; Shi, H.; Du, C. A broadband slab lens antenna formed from gradient refractive index metamaterials composed of cross-shaped cells. Microw. Opt. Techn. Lett. 2014, 56, 1124-1129. [CrossRef] 
20. Xu, W.; Xie, L.; Zhu, J.; Tang, L.; Singh, R.; Wang, C.; Ma, Y.; Chen, H.T.; Ying, Y. Terahertz biosensing with a graphene-metamaterial heterostructure platform. Carbon 2019, 141, 618-625. [CrossRef]

21. He, X.; Lin, F.; Liu, F.; Shi, W. Tunable high Q-factor terahertz complementary graphene metamaterial. Nanotechnology 2018, 29, 485205. [CrossRef] [PubMed]

22. Xia, L.; Zhang, X.; Wei, D.; Cui, H.L.; Du, C. Graphene terahertz amplitude modulation enhanced by square ring resonant structure. IEEE Photonics J. 2018, 10, 1-7. [CrossRef]

23. Gao, W.; Shu, J.; Reichel, K.; Nickel, D.V.; He, X.; Shi, G.; Vajtai, R.; Ajayan, P.M.; Kono, J.; Mittleman, D.M.; et al. High-contrast terahertz wave modulation by gated graphene enhanced by extraordinary transmission through ring apertures. Nano Lett. 2014, 14, 1242-1248. [CrossRef] [PubMed]

24. Ju, L.; Geng, B.; Horng, J.; Girit, C.; Martin, M.; Hao, Z.; Bechtel, H.A.; Liang, X.; Zettl, A.; Shen, Y.R.; et al. Graphene plasmonics for tunable terahertz metamaterials. Nat. Nanotechnol. 2011, 6, 630. [CrossRef] [PubMed]

25. Sun, F.; Xia, L.; Nie, C.; Shen, J.; Zou, Y.; Cheng, G.; Wu, H.; Zhang, Y.; Wei, D.; Yin, S.; et al. The all-optical modulator in dielectric-loaded waveguide with graphene-silicon heterojunction structure. Nanotechnology 2018, 29, 135201. [CrossRef] [PubMed]

26. Mao, Q.; Wen, Q.Y.; Tian, W.; Wen, T.L.; Chen, Z.; Yang, Q.H.; Zhang, H.W. High-speed and broadband terahertz wave modulators based on large-area graphene field-effect transistors. Opt. Lett. 2014, 39, 5649-5652. [CrossRef] [PubMed]

(C) 2019 by the authors. Licensee MDPI, Basel, Switzerland. This article is an open access article distributed under the terms and conditions of the Creative Commons Attribution (CC BY) license (http:/ / creativecommons.org/licenses/by/4.0/). 